\title{
(6) OPEN ACCESS \\ Performance characteristics of methods for quantifying spontaneous intracerebral haemorrhage: data from the Efficacy of Nitric Oxide in Stroke (ENOS) trial
}

\author{
Kailash Krishnan, ${ }^{1}$ Siti F Mukhtar, ${ }^{1}$ James Lingard, ${ }^{1}$ Aimee Houlton, ${ }^{1}$ \\ Elizabeth Walker, ${ }^{1}$ Tanya Jones, ${ }^{1}$ Nikola Sprigg, ${ }^{1}$ Lesley A Cala, ${ }^{2}$ Jennifer L Becker, ${ }^{3}$ \\ Robert A Dineen, ${ }^{4}$ Panos Koumellis, ${ }^{5}$ Alessandro Adami, ${ }^{6}$ Ana M Casado, ${ }^{7}$ \\ Philip M W Bath, ${ }^{1}$ Joanna M Wardlaw ${ }^{7}$
}

- Additional material is published online only. To view please visit the journal online (http://dx.doi.org/10.1136/ jnnp-2014-309845)

For numbered affiliations see end of article.

\section{Correspondence to} Professor Philip M W Bath, Stroke, Division of Clinical Neuroscience, University of Nottingham, Clinical Sciences Building, City Hospital campus, Hucknall Road, Nottingham NG5 1PB UK; philip.bath@ nottingham.ac.uk

Received 3 November 2014 Accepted 8 December 2014 Published Online First 9 January 2015

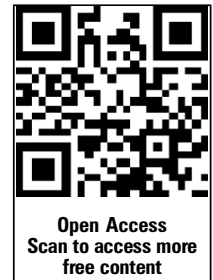

\section{CrossMark}

To cite: Krishnan $\mathrm{K}$, Mukhtar SF, Lingard J, et al. J Neurol Neurosurg Psychiatry 2015;86: 1258-1266.

\section{ABSTRACT}

Background Poor prognosis after intracerebral haemorrhage $(\mathrm{ICH})$ is related to haemorrhage characteristics. Along with developing therapeutic interventions, we sought to understand the performance of haemorrhage descriptors in large clinical trials.

Methods Clinical and neuroimaging data were obtained for 548 participants with ICH from the Efficacy of Nitric Oxide in Stroke (ENOS) trial. Independent observers performed visual categorisation of the largest diameter, measured volume using $A B C / 2$, modified $A B C / 2$, semiautomated segmentation ( $S A S)$, fully automatic measurement methods; shape, density and intraventricular haemorrhage were also assessed. Intraobserver and interobserver reliability were determined for these measures.

Results $\mathrm{ICH}$ volume was significantly different among standard $A B C / 2$, modified $A B C / 2$ and SAS: (mean) 12.8 (SD 16.3), $8.9(9.2), 12.8(13.1) \mathrm{cm}^{3}$, respectively $(p<0.0001)$. There was excellent agreement for haemorrhage volume ( $n=193)$ : $A B C / 2$ intraobserver intraclass correlation coefficient (ICC) $0.96-0.97$, interobserver ICC 0.88; modified $A B C / 2$ intraobserver ICC 0.95-0.97, interobserver ICC 0.91; SAS intraobserver ICC 0.95-0.99, interobserver ICC 0.93; largest diameter: (visual) interadjudicator ICC 0.82 , (visual vs measured) adjudicator vs observer ICC 0.71 ; shape intraobserver ICC 0.88 interobserver ICC 0.75 ; density intraobserver ICC 0.86 , interobserver ICC 0.73 . Graeb score (mean 3.53) and modified Graeb (5.22) scores were highly correlated. Using modified $A B C / 2, I C H$ volume was underestimated in regular (by $2.2-2.5 \mathrm{~cm}^{3}, p<0.0001$ ) and irregularshaped haemorrhages (by $4.8-4.9 \mathrm{~cm}^{3}, \mathrm{p}<0.0001$ ). Fully automated measurement of haemorrhage volume was possible in only $5 \%$ of cases.

Conclusions Formal measurement of haemorrhage characteristics and visual estimates are reproducible. The standard $A B C / 2$ method is superior to the modified $A B C /$ 2 method for quantifying ICH volume.

Clinical trial registration ISRCTN9941422.

\section{INTRODUCTION}

Spontaneous intracerebral haemorrhage (ICH) is usually a severe form of stroke with a high mortality rate $(\sim 50 \%)$ by the end of the first year. ${ }^{1}$ Poor functional outcome is related independently to increased age, systolic blood pressure, stroke severity and reduced level of consciousness. Radiologically, haemorrhage size, haemorrhage expansion, evidence of continuing bleeding (eg, 'spot sign' on CT angiography) ${ }^{2}{ }^{3}$ and presence of intraventricular haemorrhage (IVH) are all also associated with poor outcome. ${ }^{45}$ The combination of ICH volume and initial Glasgow Coma scale was reported to be the strongest independent predictor of 30-day outcome. ${ }^{1}$ Hence, reliable measurement of haemorrhage size is an important component of early clinical management and for stratification in clinical trials.

Although any effective intervention will ultimately need to show an effect on functional outcome (eg, using the modified Rankin Scale $(\mathrm{mRS}))^{6}$ in phase III trials, earlier developments will need to study the effect of treatment on a surrogate measure. Where the intervention aims to limit haemorrhage expansion, accurate, reproducible and validated measurement of haemorrhage size (thereby allowing measurement of the change in volume) will be critical. Hence, phase II trials of blood pressure lowering and haemostatic interventions have utilised measurement of haemorrhage expansion as a key primary outcome. ${ }^{78}$

There are multiple methods for measuring haemorrhage volume on a CT scan, ranging from qualitative visual estimation to computerised automatic measurement. Visual approaches (ie, visual size categorisation based on the largest diameter) are quick while computerised methods might provide a continuous volume measure but require access to good quality electronic image files. A common approach is the $\mathrm{ABC} / 2$ method, ${ }^{9}$ which has been modified such that haemorrhage slices are excluded if the area is $<25 \%$ of the largest slice and counted as half if it is $25-75 \%$ of the largest slice. ${ }^{10}$ Alternatively, semiautomatic or fully automatic computerised methods may be used, usually based on manually outlining or thresholding areas or volumes within regions of interest (ROI). The relative advantages and disadvantages of these approaches reflect the balance between measurement time, type of data required, accuracy and 
availability of computer workstations, and sample size, as studied in part. ${ }^{11-14}$ Assessment of additional properties of haemorrhage, such as shape and density ${ }^{15}$ and extension into the ventricles or subarachnoid space, may also be useful. ${ }^{16} 17$

In this study, we assessed and compared methods for measuring haemorrhage size, shape and density and IVH size. The data come from the 'Efficacy of Nitric Oxide in Stroke' (ENOS) trial, ${ }^{18}$ which assessed the management of blood pressure in acute stroke and included patients with ICH.

\section{METHODS}

\section{Patients}

Data came from the prospective international multicentre ENOS trial, a study of blood pressure management in patients with acute ischaemic stroke or ICH, and high blood pressure. ${ }^{18}$ The trial had regulatory and ethics approvals in each participating country, and all patients gave written consent, or a relative provided proxy consent if the patient lacked capacity. The main inclusion criteria were acute stroke with motor deficit, systolic blood pressure of 140-220 $\mathrm{mm} \mathrm{Hg}$, and treatment could start within $48 \mathrm{~h}$ of onset. Patients were recruited from 173 sites in 23 countries across five continents. Following the entry of baseline demographic and clinical data into a secure internet-fronted database, patients were randomised to transdermal glyceryl trinitrate $(5 \mathrm{mg}$ ) versus none for 7 days; those taking antihypertensive agents immediately prior to their stroke were also randomised to continue versus stop these, again for 7 days.

Participants had a baseline CT or MRI brain scan as part of clinical care, usually before randomisation. Where possible, a second (research) CT or MRI scan was performed at day $7 \pm 1$ (end-of-treatment). DICOM, JPEG, PNG or GIF files are sent to the coordinating centre. Any scans sent on film were digitised using a VICOM digitiser (VIDAR Diagnostic PRO Advantage, USA). The following study assesses only those patients with a CT scan. For each patient, images were received with the thinnest slices provided by CT scan machines using standardised protocols in the recruiting centres. However, during the course of the trial, more volumetric images were made available because of advances in imaging technology.

\section{Scan adjudication}

Complete CT scan series were made available for assessment by a group of seven adjudicators comprising accredited neuroradiologists or neurologists trained in CT brain imaging assessment in stroke (http://www.neuroimage.co.uk/sirs, with coordination by JMW). ${ }^{19}$ Images were viewed over the web, blind to all clinical and treatment information except for patient age, time since onset of stroke and side of brain symptoms. Responses were entered directly into the trial database via a web-based response form. Collected information included whether the patient had an ICH and its location; an estimate of its size (sorted into ordered categories based on the longest diameter in any plane): $<3,3-4.9,5-8,>8 \mathrm{~cm}$; and the presence of other qualitative findings (mass effect, ${ }^{19}$ atrophy, ${ }^{19}$ white matter disease, ${ }^{20}$ old infarct or haemorrhage) using validated scoring methods.

\section{Scan haemorrhage quantification}

Scans were visualised and analysed using OsiriX for Mac (V.3, 32 bit, http://www.osirix-viewer.com ${ }^{21}$ on a 26 inch Apple iMac. Two trained observers (KK, SFM) measured baseline ICH volumes of 193 patients using $\mathrm{ABC} / 2$, modified $\mathrm{ABC} / 2$, semiautomatic segmentation (SAS) and automatic volume calculation (AVC, using three-dimensional (3D) rendering of a stack of two-dimensional slices). Both observers assessed scans blinded to each other's data, and repeated a proportion of the scans (47 and 34 scans for KK and SFM, respectively) at a different time blinded to their original measurements. Thus, intraobserver and interobserver variation could be estimated. For the remaining 355 patients, one observer (KK) calculated $\mathrm{ICH}$ volumes using all four methods.

The $\mathrm{ABC} / 2$ method requires measurement of the longest diameter of the haemorrhage in the axial plane ('A'), longest axis at $90^{\circ}$ to 'A' in the axial plane ('B'), and the number of slices (' $\mathrm{C}$ ') showing the haemorrhage (noting that slice thickness may vary through the scan). ' $\mathrm{C}$ ' is the product of slice thickness and number of slices showing any haemorrhage. Volume is calculated as $\mathrm{A} \times \mathrm{B} \times \mathrm{C} / 2 .{ }^{9}$ In the modified $\mathrm{ABC} / 2$ method, the area of haemorrhage seen on a slice has to be at least $25 \%$ of the haemorrhage area seen on the largest slice if the slice is to be considered in ' $\mathrm{C}$ '; if the area is more than $75 \%$ of the largest area, the slice counts as 1 , and 0.5 if the measured value of the area is between $25 \%$ and $75 \% .^{10}$

For SAS and AVC, it was possible to use thresholds so that the haemorrhage ROI was measured within certain segmentation parameters. Upper and lower attenuation values (typically 40$80 \mathrm{HU}$ ) of haemorrhage were established manually by sampling from the haemorrhage and normal brain. Where the threshold did not exclude non-haemorrhage areas (as with the pineal gland, calcified choroid plexus, bone) in close proximity with the haemorrhage, the haemorrhage boundary was manually edited. The area of each slice that was included within these parameters was measured, added together and multiplied by scan thickness to obtain the SAS volume. AVC used an Osirix '3D growing region (entire series)' method after selecting a threshold part of the haemorrhage near to its centre. With all methods, IVH blood was included in the final ICH volume.

Additionally, four haemorrhage characteristics (area, perimeter, mean attenuation and SD of attenuation) were recorded for the haemorrhage from the slice with the largest area of haemorrhage. Haemorrhage shape and density indices were then calculated as:

$$
\begin{gathered}
\text { Shape index [ref. 22] }=\text { perimeter }^{2} / 4 \Pi \times \text { area } \\
\text { Density index }[\text { ref. } 23]=S D / \text { mean }(\text { ie }, \text { coefficient of variation })
\end{gathered}
$$

The divergence in visual appearance of haemorrhage shape and density were also assessed used an ordered categorical scale (1-5) where for an extra lesion edge or degree of density variation an additional point was given on the shape scale or density scale. ${ }^{15}$ Similarly, intraventricular blood volume was assessed visually using the Graeb score and modified Graeb ordered categorical scales. ${ }^{16} 17$ The Graeb score is calculated by scoring the amount of blood in the lateral ventricles separately (score of $1=$ trace of blood to a maximum of $4=$ ventricle full of blood and expanded) and adding this to the scores for the third and fourth ventricles separately $(1=$ blood present and $2=$ filled with blood and expanded) to derive a maximum score of 12 . The modified Graeb score allocates scores for separate ventricular compartments ( $1 \leq 25 \%$ filled with blood to $4 \geq 75 \%$ filled with blood) to reflect selective regional accumulation of blood and an extra one point for expansion of each ventricle. The maximum score is 32 .

Intraobserver and interobserver reliability was determined for visual categorisation of shape and density. In addition, intraobserver reliability was assessed for measured shape, density index, Graeb, modified Graeb and IVH volume over different reading sessions, separated by a minimum of 14 days. All adjudication and haemorrhage measurements were made blind to baseline and follow-up clinical information, other imaging and treatment assignment. 


\section{Statistical analysis}

Data are shown as number (\%), median (IQR) or mean (SD). Measurement of intraobserver and interobserver variability was assessed using the intraclass correlation coefficient (ICC). ${ }^{24} \mathrm{~A}$ probability value of $<0.05$ was considered statistically significant. Analyses were performed using SPSS (V.21) and checked with Medistat running on an Apple Mac.

Altogether, 629 patients with ICH were enrolled into ENOS. ${ }^{25} 26$ Of these, 548 patients had CT-confirmed ICH and a baseline scan available for measurement; 81 other patients either had ICH diagnosed on MRI or had no CT scan available, and these were excluded. Patient demographic and clinical details are shown in table 1 . The mean age of the 548 patients in the present analysis was 67 (SD 12) years, 66\% of patients were male, mean baseline blood pressure was 171 (19)/92 (13) $\mathrm{mm} \mathrm{Hg}$, and the median time from onset of ictus to performing neuroimaging was 4.5 (IQR 5.6) hours. When adjudicated visually by experts, $63 \%$ of haematomas were located in the middle cerebral artery territory (table 1); most haemorrhages caused a mass effect (86\%) and many patients had leukoaraiosis $(66 \%)$ and/or a previous stroke lesion (49\%) present on their scans. The most frequent visually assessed haemorrhage length category was $3-5 \mathrm{~cm}(224,41.3 \%)$, closely followed by $<3 \mathrm{~cm}(220,40.6 \%)$ with much fewer larger haemorrhages. The mean measured haemorrhage length was $3.4 \mathrm{~cm}$ (longest diameter; table 1).

\section{ICH volume}

$\mathrm{ICH}$ volume was significantly different between $\mathrm{ABC} / 2$, modified $\mathrm{ABC} / 2$ and SAS: 12.8 (mean) (SD 16.3), 8.9 (9.2), 12.8 (13.1) $\mathrm{cm}^{3}$, respectively $(\mathrm{p}<0.0001)$ (table 2$)$. The ICC was 'excellent' at $0.84-0.96$ when comparing the observers' measurements for each of $\mathrm{ABC} / 2$, modified $\mathrm{ABC} / 2$ and SAS (table 2). The observers found haemorrhage volume to be larger, by an average of $2.7-4 \mathrm{~cm}^{3}$ with standard $\mathrm{ABC} / 2$, as compared to modified $\mathrm{ABC} / 2$, and $1.4-4 \mathrm{~cm}^{3}$ smaller with $\mathrm{SAS}$ as compared to modified $\mathrm{ABC} / 2$. As the mean $\mathrm{ICH}$ value increased, the difference between $\mathrm{ICH}$ volume measured by modified $\mathrm{ABC} / 2$ versus the other two methods increased (figures 1 and 2), but no significant difference was observed between standard ABC/2 and SAS (table 2; figure 3). The slope of the best-fit regression line for the increasing difference between modified $\mathrm{ABC} / 2$ versus $\mathrm{ABC} / 2$ was -0.44 , and -0.36 for modified $\mathrm{ABC} / 2$ versus $\mathrm{SAS}$ (both $\mathrm{p}<0.0001$ ) (figures 1 and 2).

There was good intraobserver agreement for measurements assessed on $34-47$ scans $(5-10 \%$ of the total of 548$)$. For $\mathrm{ABC} / 2$, modified $\mathrm{ABC} / 2$ and SAS, the intraobserver ICC was 'excellent', ranging between 0.97-0.98, and 0.95-0.99, respectively (table 3).

There was excellent interobserver agreement (ICC) based on 193 scans at 0.88 for $\mathrm{ABC} / 2,0.91$ for modified $\mathrm{ABC} / 2$ and 0.93 for SAS (table 4). Both $\mathrm{ABC} / 2$ and modified $\mathrm{ABC} / 2$ showed excellent correlation with SAS $(p<0.0001$, see online supplementary figures S1-S3). Only 23 of 548 scans were amenable to analysis using the fully AVC method as the software was unable to handle the scan image with varying slice thickness; this approach was therefore ignored in further analyses.

Across the $\mathrm{ICH}$ visual size categories, $\mathrm{ICH}$ volumes calculated by modified $\mathrm{ABC} / 2$ were significantly smaller compared to SAS (figure 4). As the $\mathrm{ICH}$ size category increased, the difference between modified $\mathrm{ABC} / 2$ and SAS also increased. By comparison, there was no significant difference between ICH volumes

\section{RESULTS}

Table 1 Baseline demographic, clinical and neuroradiological factors in 548 patients with primary intracerebral haemorrhage in the ENOS trial

\begin{tabular}{|c|c|}
\hline Variable & Data \\
\hline \multicolumn{2}{|l|}{ Demographics } \\
\hline Age (years) & $67.9(12.1)$ \\
\hline Sex, male $(\%)$ & $360(65.7)$ \\
\hline \multicolumn{2}{|l|}{ Country, UK (\%) } \\
\hline Asia & $117(21.4)$ \\
\hline Europe & $67(21.4)$ \\
\hline Other (Africa, Australasia and North America) & $42(7.7)$ \\
\hline UK & $322(58.8)$ \\
\hline \multicolumn{2}{|l|}{ Clinical findings } \\
\hline Premorbid modified Rankin Scale=0 $(\%)$ & $418(76.3)$ \\
\hline Previous stroke (\%) & $69(12.6)$ \\
\hline Prior antihypertensive medication use (\%) & $227(41.4)$ \\
\hline Prior history of high BP (\%) & $341(62.2)$ \\
\hline Diabetes mellitus (\%) & $67(12.2)$ \\
\hline Ischaemic heart disease (\%) & $56(10.2)$ \\
\hline Atrial fibrillation (\%) & $30(6.5)$ \\
\hline Total anterior circulation syndrome (\%) & $195(35.6)$ \\
\hline SSS (/58) & $30.1(12.3)$ \\
\hline National Institutes of Health Stroke Scale (/42) & $12.8(5.3)$ \\
\hline Systolic BP (mm Hg) & $171.6(19.3)$ \\
\hline Diastolic BP (mm Hg) & $92.2(13.3)$ \\
\hline Heart rate $(\mathrm{bpm})$ & $77.7(14.5)$ \\
\hline Time, stroke to neuroimaging (h) & $4.5[11.6]$ \\
\hline \multicolumn{2}{|l|}{ Adjudicated CT scan findings } \\
\hline \multicolumn{2}{|l|}{ Location of haemorrhage (\%) } \\
\hline MCA & $346(63.1)$ \\
\hline ACA & $22(4.0)$ \\
\hline PCA & $5(0.9)$ \\
\hline $\mathrm{MCA}+\mathrm{ACA}$ & $2(0.4)$ \\
\hline Borderzone & $14(2.6)$ \\
\hline Lacunar (ie, small subcortical) stroke & $142(25.9)$ \\
\hline Brainstem and/or cerebellum & $16(2.9)$ \\
\hline Leukoariosis & $362(66.1)$ \\
\hline Lesion mass effect (minimal to extreme swelling) & $470(85.9)$ \\
\hline Previous stroke lesion & $270(49.3)$ \\
\hline IVH & $141(25.7)$ \\
\hline \multicolumn{2}{|l|}{ Intracerebral haematoma size category (\%) } \\
\hline$<3 \mathrm{~cm}$ & $220(40.6)$ \\
\hline 3 to $<5 \mathrm{~cm}$ & $224(41.3)$ \\
\hline 5 to $8 \mathrm{~cm}$ & $87(16.1)$ \\
\hline$>8 \mathrm{~cm}$ & $11(2.0)$ \\
\hline \multicolumn{2}{|l|}{ Measured CT scan findings } \\
\hline Volume, $A B C / 2\left(\mathrm{~cm}^{3}\right)$ & $12.77(16.32)$ \\
\hline Longest diameter $(\mathrm{cm})$ & $3.38(1.4)$ \\
\hline \multicolumn{2}{|l|}{ With IVH, n=141 } \\
\hline Graeb score $(/ 12)^{16}$ & $3.52(2.4)$ \\
\hline Modified Graeb score $(/ 32)^{17}$ & $5.19(4.7)$ \\
\hline \multicolumn{2}{|l|}{ Without IVH, n=407 } \\
\hline Shape $(15)^{15}$ & $3.00(1.4)$ \\
\hline Shape index ${ }^{22}$ & $1.22(1.1)$ \\
\hline Density $(/ 5)^{15}$ & $2.54(1.3)$ \\
\hline Density index ${ }^{23}$ & $0.19(0.1)$ \\
\hline \multicolumn{2}{|c|}{$\begin{array}{l}\text { Data are number (\%), median (semiquartile range), or mean (SD). } \\
\text { NIHSS calculated from SSS scores range from } 0 \text { (comatose with quadriplegia) to } 58 \\
\text { (normal neurological status). } \\
\text { ACA, anterior cerebral artery territory; BP, blood pressure; ENOS, Efficacy of Nitric } \\
\text { Oxide in Stroke; IVH, intraventricular haemorrhage; MCA, middle cerebral artery } \\
\text { territory; NIHSS, the National Institutes of Health Stroke Scale; PCA, posterior cerebral } \\
\text { artery territory; SAS, semiautomatic segmentation; SSS, Scandinavian Stroke Scale. }\end{array}$} \\
\hline
\end{tabular}


Table 2 Comparison by two observers of different methods for measuring haemorrhage volume $\left(\mathrm{cm}^{3}\right)$ on $C T$ scans: $A B C / 2$ versus modified $A B C / 2$ versus $S A S$

\begin{tabular}{|c|c|c|c|c|}
\hline Haematoma volume & & Difference & p Value & ICC \\
\hline \multicolumn{5}{|l|}{$\begin{array}{l}\text { Observer } 1 \\
n=548\end{array}$} \\
\hline $\begin{array}{l}A B C / 2 \\
12.77(16.32)\end{array}$ & $\begin{array}{l}\text { Modified ABC/2 } \\
8.90(9.21)\end{array}$ & 3.96 & $<0.0001$ & 0.84 \\
\hline $\begin{array}{l}A B C / 2 \\
12.77(16.32)\end{array}$ & $\begin{array}{l}\text { SAS } \\
12.76(13.06)\end{array}$ & -0.01 & 1.00 & \\
\hline $\begin{array}{l}\text { SAS } \\
12.76(13.06)\end{array}$ & $\begin{array}{l}\text { Modified ABC/2 } \\
8.90(9.21)\end{array}$ & 3.97 & $<0.0001$ & \\
\hline \multicolumn{5}{|l|}{$\begin{array}{l}\text { Observer } 2 \\
n=193\end{array}$} \\
\hline $\begin{array}{l}A B C / 2 \\
12.05(12.40)\end{array}$ & $\begin{array}{l}\text { Modified ABC/2 } \\
9.70(10.05)\end{array}$ & 2.67 & $<0.0001$ & 0.96 \\
\hline $\begin{array}{l}A B C / 2 \\
12.05(12.40)\end{array}$ & $\begin{array}{l}\text { SAS } \\
11.08(11.38)\end{array}$ & 0.05 & 0.89 & \\
\hline $\begin{array}{l}\text { SAS } \\
11.08(11.38)\end{array}$ & $\begin{array}{l}\text { Modified ABC/2 } \\
9.70(10.05)\end{array}$ & 1.38 & $<0.0001$ & \\
\hline
\end{tabular}

measured by standard $\mathrm{ABC} / 2$ and SAS when compared by $\mathrm{ICH}$ visual size categorisation (figure 4).

There was good agreement between the adjudicators across $\mathrm{ICH}$ visual size categories $(\mathrm{n}=47$, ICC $0.82, \mathrm{p}<0.001)$ (see online supplementary table I) using the ordered categorical scale $(<3,3-4.9,5-8,>8 \mathrm{~cm})^{19}$ and strong agreement between the visual size category and the observer's largest measured diameter in the axial plane 'A' (ICC $0.71, \mathrm{p}<0.001$ ) (see online supplementary table II).

\section{ICH volume and shape}

The most common shape was irregular (64\%), followed by regular ICH. Small ICHs $\left(5.5-8.06 \mathrm{~cm}^{3}\right)$ were more regularly shaped than larger haemorrhages $(\mathrm{p}<0.0001)$ (table 5). Using modified $\mathrm{ABC} / 2$, haemorrhage volume was significantly lower for regular-shaped haemorrhages when compared with $\mathrm{ABC} / 2$ (by $2.2 \mathrm{~cm}^{3}$ ) and SAS (by $2.4 \mathrm{~cm}^{3}$ ) (table 5 and figure 5); the difference was greater with larger irregular-shaped haemorrhages (between standard $\mathrm{ABC} / 2$ and modified $\mathrm{ABC} / 2$ by $4.8 \mathrm{~cm}^{3}$ and SAS and modified $\mathrm{ABC} / 2$ by $4.9 \mathrm{~cm}^{3}$ ). When compared by shape, ICH volume calculated by standard $\mathrm{ABC} / 2 \mathrm{did}$ not differ from that measured by SAS (figure 5).

\section{ICH shape and density in patients with no IVH}

Intra-agreements and interagreements were both 'good' for visual assessments of haemorrhage shape: intraobserver ICC 0.88 (see online supplementary table III), interobserver ICC $0.75(n=47)$ (see online supplementary table IV); and density: intraobserver ICC 0.86 , interobserver ICC 0.73. Intra-observer ICC was 0.53 for calculated shape index and 0.86 for density index (see online supplementary table III).

\section{IVH volume and severity}

Intraobserver agreement for assessment of IVH volume on baseline scans from 49 patients was excellent (ICC 0.98-0.99) whether using the Graeb score, modified Graeb score or SAS (see online supplementary table III). The Graeb and modified Graeb scores were highly correlated with each other $(\mathrm{rs}=0.88$, $\mathrm{p}<0.01$ ). Both were also highly correlated with measured IVH volume using the SAS method: Graeb, $(\mathrm{rs}=0.73, \mathrm{p}<0.01)$; modified Graeb ( $\mathrm{rs}=0.72, \mathrm{p}<0.01)$.

Comparison of ICH volume by $A B C / 2$, modified $A B C / 2$ to $S A S$

We performed a non-systematic review to compare our study with previously published work comparing $\mathrm{ABC} / 2$, modified $\mathrm{ABC} / 2$ and SAS volume measures in $\mathrm{ICH}^{10-14}{ }^{27-30}$ (see online supplementary figures S4-S7).

Some studies used the standard $\mathrm{ABC} / 2$ formula while others used variations of it; ${ }^{10}{ }^{11}$ Gebel et $a l^{11}$ used the central haemorrhage slice to measure the largest diameter 'A' and found higher volumes computed by $\mathrm{ABC} / 2$ when compared to SAS. With regard to shape, three studies studied errors in warfarin-related haemorrhage due to higher frequencies of irregular shape. ${ }^{12-14}$
Figure 1 The Bland-Altman plot for assessment of variation in estimating haematoma volume between modified $A B C / 2$ and standard $A B C / 2(n=548)$, $r^{2}=0.64, p<0.0001$. The continuous and dotted lines represent the regression lines. The slope of the best-fit regression line gradient was $-0.44(p<0.0001)$.

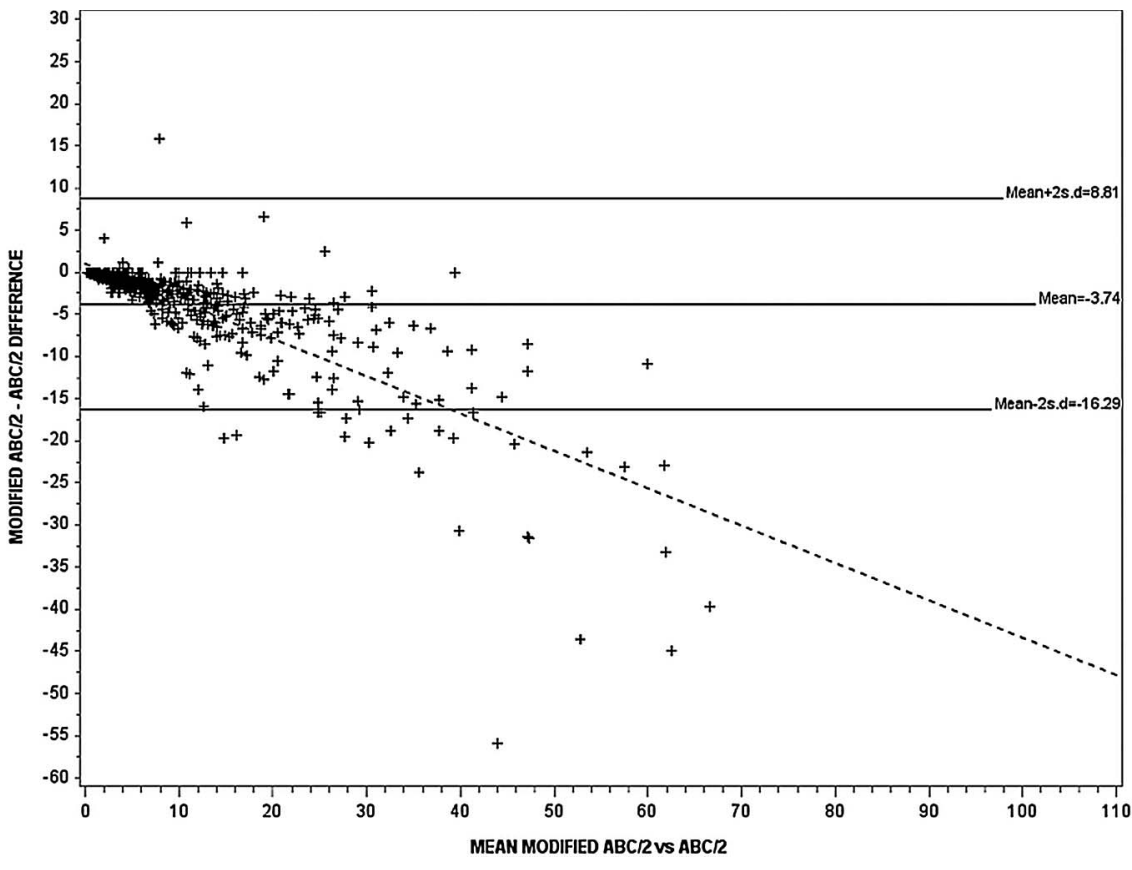


Figure 2 The Bland-Altman plot for assessment of variation in estimating haematoma volume between modified $A B C / 2$ and semiautomatic segmentation $(n=548), r^{2}=0.45$, $p<0.0001$. The continuous and dotted lines represent the regression lines. The slope of the best-fit regression line was $-0.36(p<0.0001)$.

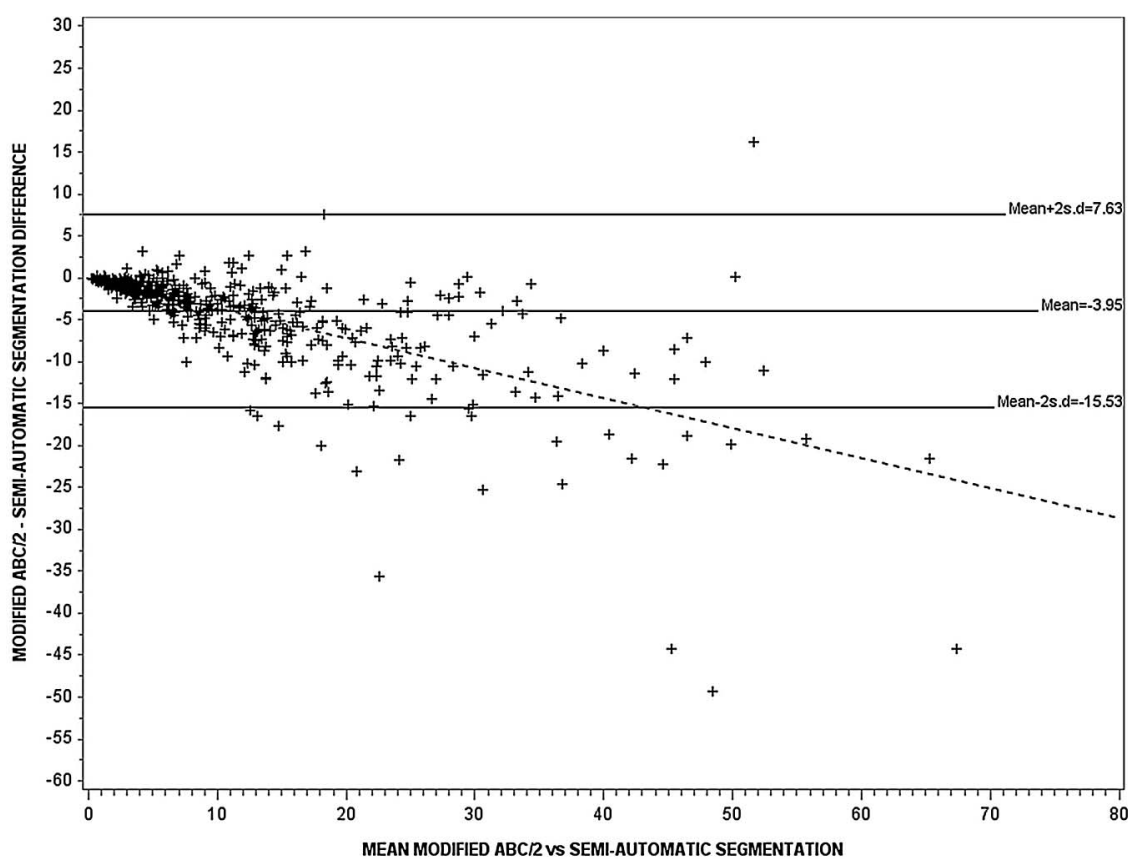

Huttner et $a l^{12}$ showed that volumes of regular shaped haematomas using $\mathrm{ABC} / 2$ were significantly increased in regular and irregular shaped haemorrhages. However, the large slice thickness used during CT image acquisition in this study may have produced significant errors during calculation. ${ }^{12}$

This review comparing all three methods showed that the $\mathrm{ABC} / 2$ formula tended to marginally overestimate the $\mathrm{ICH}$ volume, but there was no absolute significant difference compared to SAS (see online supplementary figure S4); when assessed by variability in haemorrhage shape, the results follow the same sequence (see online supplementary figure S5). By comparison, ICH volumes computed using modified $\mathrm{ABC} / 2$ are significantly smaller when compared with SAS; the difference is greater as the haemorrhage sizes became larger and more irregular (see online supplementary figures S6 and S7).

\section{DISCUSSION}

To the best of our knowledge, this is the first study that compares several methods for assessing volume of spontaneous ICH and IVH volume on CT scanning and tests the effect of haematoma shape and regularity on these measures. The major findings are as follows: (1) Agreement within and between observers was excellent for measures of haemorrhage volume (ABC/2, modified $\mathrm{ABC} / 2$, SAS) and other haemorrhage parameters (visual categorisation by maximum length, description of shape, density and IVH); (2) haemorrhage volumes measured by modified $\mathrm{ABC} / 2$ were significantly lower than those measured by $\mathrm{ABC} / 2$ and SAS planimetry; the discrepancy increased as the haematoma size became larger; (3) larger haemorrhages were significantly more irregular in shape; (4) agreement was good between visual size categorisation and measured computerised
Figure 3 The Bland-Altman plot for assessment of variation in estimating haematoma volume $(n=548)$ using $A B C / 2$ and semiautomatic segmentation $(n=548), r^{2}=0.03$, $p<0.0001$. The continuous and dotted lines represent the regression lines. The slope of the best-fit regression line was $0.02(p<0.0001)$.

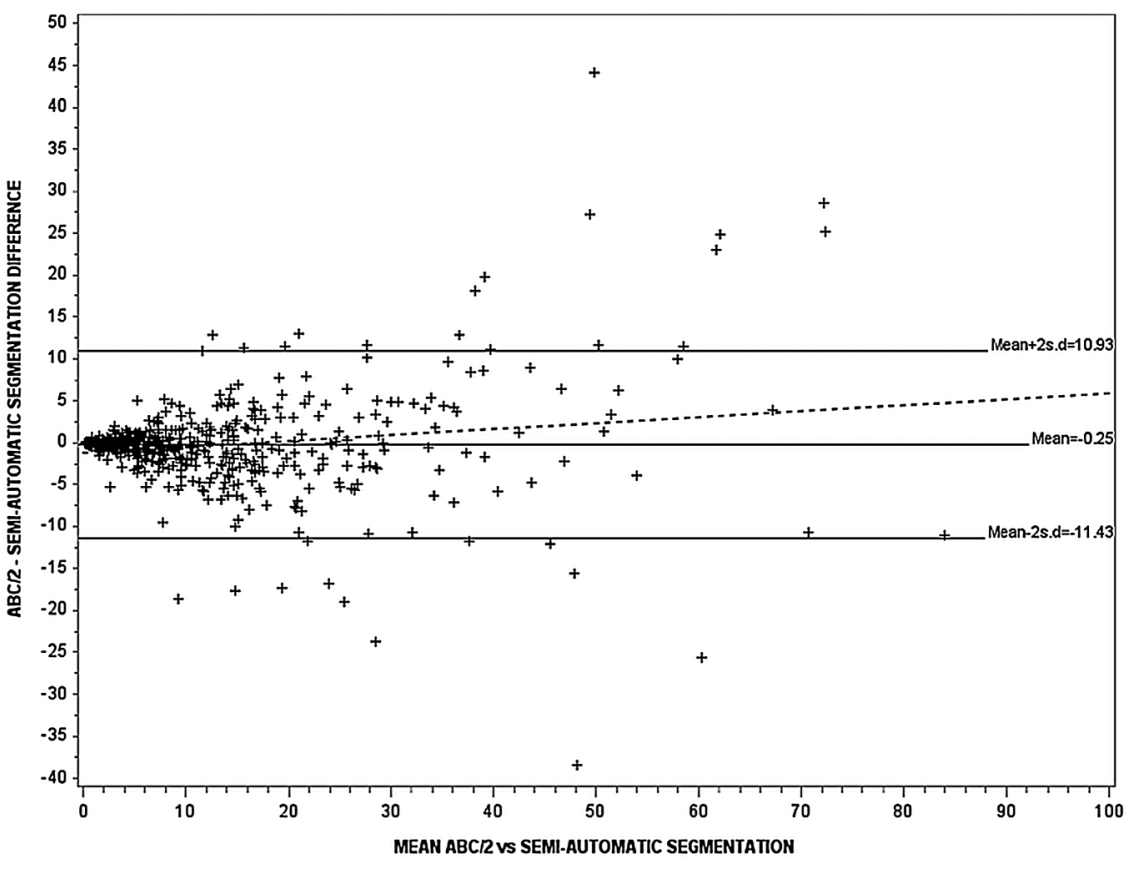


Table 3 Intraobserver comparison for two observers of haemorrhage size ( $n=47,34$ scans, respectively) on baseline CT scans from patients with ICH

\begin{tabular}{|c|c|c|c|c|c|c|c|c|}
\hline & & & Difference (p) & ICC & & & Difference $(p)$ & ICC \\
\hline Observer & 1 & & & & 2 & & & \\
\hline Measurement & 1 & 2 & & & 1 & 2 & & \\
\hline Modified $A B C / 2\left(\mathrm{~cm}^{3}\right)$ & $8.67(8.34)$ & $9.10(8.34)$ & $0.43(0.80)$ & 0.97 & $16.88(14.60)$ & $16.90(14.63)$ & $0.016(0.97)$ & 0.95 \\
\hline $\mathrm{ABC} / 2\left(\mathrm{~cm}^{3}\right)$ & $10.42(10.28)$ & $10.98(10.79)$ & -0.55 & 0.97 & $20.97(19.32)$ & $20.15(17.98)$ & $0.82(0.39)$ & 0.96 \\
\hline SAS $\left(\mathrm{cm}^{3}\right)$ & $11.11(10.38)$ & $11.69(10.99)$ & $0.58(0.80)$ & 0.98 & $19.41(15.32)$ & $19.42(15.51)$ & $-0.11(0.87)$ & 0.99 \\
\hline Diameter (A) (cm) & $3.41(1.28)$ & $3.37(1.31)$ & $0.04(0.89)$ & 0.98 & $3.89(1.48)$ & $4.01(1.45)$ & $-0.12(0.43)$ & 0.96 \\
\hline
\end{tabular}

measurement of maximum haemorrhage diameter; (5) attempts to perform a fully automated volume measurement method failed in more than $95 \%$ of patients, as the software was unable to work with scans in which slice thicknesses varied.

The sources of error that effect variation between $\mathrm{ABC} / 2$ and SAS merit consideration. First, the largest diameter of haemorrhage was measured in the axial plane but this may not be the largest ICH diameter, which may also have contributed to differences between 'A' and the visual size categorisation. Sucu et $a l^{31}$ suggested using the maximum length and width not necessarily on the same slice and found better correlation between ABC/2 and SAS. However, this adaptation applies only to chronic subdural haematomas which differ from spontaneous haemorrhages by extending to the cranial vault and being crescentic. ${ }^{31}$ Second, when using $\mathrm{ABC} / 2$, the scan plane is measured through $\mathrm{AB} / 2$, the formula for the area of a triangle. However, a triangle is not necessarily the most appropriate description of a haematoma. Last, standard $\mathrm{ABC} / 2$ approximates the haematoma volume as an ellipsoid with all three axes extending in three perpendicular directions ${ }^{9}$; to compensate for the varying slice thickness, ' $\mathrm{C}$ ' is derived by multiplying the number of slices of which the haematoma is seen by the slice thickness in centimetres. For the modified $\mathrm{ABC} / 2$, ' $\mathrm{C}$ ' does not include slices if the area of the haemorrhage is less than $25 \%$ of the largest area, ${ }^{10}$ an approach that has no theoretical justification. Hence, this method does not estimate all three Cartesian coordinates of the ellipsoid. Since the modified ABC/2 method underestimated the $\mathrm{ICH}$ volume (by $2-4 \mathrm{~cm}^{3}$ ), and small differences in volume equate to the variation in outcome, ${ }^{32}$ the modified $\mathrm{ABC} / 2$ method cannot be recommended. Further, modern CT scanners provide thin slices and the ability to directly measure ' $C$ ', thereby eliminating the need for approximating slice areas. ${ }^{33} 34$

Although computer-assisted methods are considered the gold standard for volume measure,,$^{1} 11 \quad 12 \quad 35$ the SAS method was time consuming and at times technically challenging. For

Table 4 Interobserver comparison for two (observer 1 and 2; $\mathrm{n}=193$ scans) of haemorrhage volume $\left(\mathrm{cm}^{3}\right)$ on CT scans from patients with intracerebral haemorrhage $(\mathrm{ICH})$

\begin{tabular}{|c|c|c|c|c|}
\hline & \multicolumn{2}{|l|}{ Observer } & \multirow[b]{2}{*}{ Difference $(\Delta)$} & \multirow[b]{2}{*}{ ICC } \\
\hline & 1 & 2 & & \\
\hline $\mathrm{ABC} / 2$ & $10.58(10.20)$ & $12.05(12.40)$ & -1.40 & 0.88 \\
\hline Modified $A B C / 2$ & $8.42(8.53)$ & $9.70(10.05)$ & -1.3 & 0.91 \\
\hline SAS & $12.02(12.05)$ & $11.08(11.38)$ & 0.95 & 0.93 \\
\hline
\end{tabular}

instance, it was difficult to set segmentation parameters to identify a haemorrhage that was adjacent to the bone; and the threshold may miss an area of haemorrhage. Additionally, the threshold method will not account for oedema associated with the ICH, which increases the mass effect; thus, the spaceoccupying effect of the ICH may be much larger than its measured hyperattenuated area. As segmentation is semiautomatic, it is feasible to adjust manually for errors if they are apparent, although any adjustment may be subjective. All images require visual checking and manual correction; failure to do this would result in erroneous measurements. The problem is compounded by more irregular spontaneous haemorrhages, for example, due to amyloid, in anticoagulant-associated bleeds and in traumatic haematomas, where typically lesions are irregular, of varied attenuation and often next to the bone.

In this series of patients with spontaneous $\mathrm{ICH}$, a high proportion $(64 \%)$ of irregular-shaped haemorrhages were found. The effectiveness of the $\mathrm{ABC} / 2$ method has been validated in regular, oval-shaped haemorrhages but researchers have doubted its accuracy in complex irregular haemorrhages (eg, as seen with warfarin or in large or amyloid-related haemorrhage) and those with intraventricular extension. ${ }^{12}{ }^{35}$ It has been suggested that adjusting the denominator from 2 to 3 in $\mathrm{ABC} / 2$ in irregularly shaped haematomas may produce more accurate measures, but this concept is yet to be supported. ${ }^{12}{ }^{14}$ Combining the present and published findings, ${ }^{29}$ it may be postulated that as the haemorrhage became more irregular, the surface area and volume changes more than the largest diameter. Hence, the area of the largest haemorrhage slice may be more representative of the total haemorrhage size than its diameter. As a result, the true

Table 5 Comparison of volumes $\left(\mathrm{cm}^{3}\right)$ using $A B C / 2$, modified $A B C / 2$ and semiautomated segmentation (SAS) $(n=548)$ by haematoma shape

\begin{tabular}{|c|c|c|c|c|}
\hline & $\begin{array}{l}\text { Haematoma } \\
\text { volume }\end{array}$ & & $\begin{array}{l}\text { Difference } \\
(\Delta)\end{array}$ & $p$ value \\
\hline $\begin{array}{l}\text { Regular }(1,2) \\
n=198\end{array}$ & $\begin{array}{l}A B C / 2 \\
8.06(10.43) \\
A B C / 2 \\
8.06(10.43) \\
\text { SAS } \\
7.77(8.75)\end{array}$ & $\begin{array}{l}\text { Modified ABC/2 } \\
5.56(6.31) \\
\text { SAS } \\
7.77(8.75) \\
\text { Modified ABC/2 } \\
5.56(6.31)\end{array}$ & $\begin{array}{l}2.51 \\
0.29\end{array}$ & $\begin{array}{c}<0.0001 \\
0.20 \\
<0.0001\end{array}$ \\
\hline $\begin{array}{l}\text { Irregular } \\
(3,5) \\
n=350\end{array}$ & $\begin{array}{l}A B C / 2 \\
15.48(18.37) \\
A B C / 2 \\
15.48(18.37) \\
\text { SAS } \\
15.62(14.22)\end{array}$ & $\begin{array}{l}\text { Modified ABC/2 } \\
10.66(10.07) \\
\text { SAS } \\
15.62(14.22) \\
\text { Modified ABC/2 } \\
10.66(10.07)\end{array}$ & $\begin{array}{r}4.81 \\
-0.14 \\
4.96\end{array}$ & $\begin{array}{c}<0.0001 \\
0.82 \\
<0.0001\end{array}$ \\
\hline
\end{tabular}

Data are mean (SD), difference $(\Delta)$ in volume and $p$ value. 
Figure 4 Box plots of intracerebral haemorrhage $(\mathrm{ICH})$ volumes $(n=548)$ by visually estimated size and corresponding volume measured by $A B C / 2$, modified $A B C / 2$ and semiautomatic segmentation (SAS). The difference between modified $A B C /$ 2 versus standard $A B C / 2$ and modified $A B C / 2$ versus $S A S$ increases as the size category increases and is present in all four size categories $(<3 \quad(p<0.0001)$, 3-5 ( $p<0.001), 5-8(p<0.0001)$ and $>8(p<0.0001))$. There was no significant difference between standard $A B C / 2$ and $S A S$ in all four size categories.

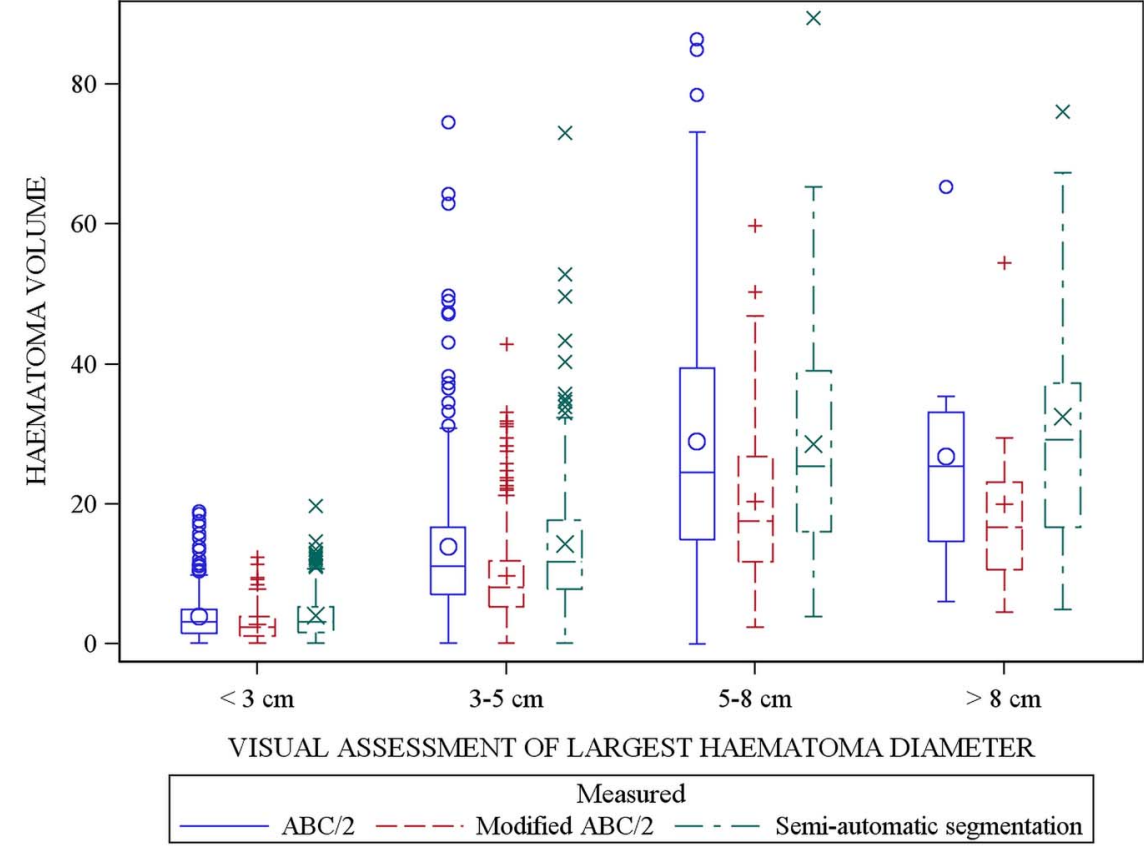

volume of an irregular haemorrhage may be better estimated using SAS. This finding is important since irregular shape haemorrhages are more likely to expand and affect morbidity and mortality. $^{15}$

Our study is novel in design as it compares two commonly used methods $\mathrm{ABC} / 2$ and the modified formula (25\%/75\% distinction) with computer-assisted SAS and pragmatic visual scoring. Moreover, haemorrhage shape was incorporated in the analysis so that the source of error within each individual measurement method was assessed further. Our results show good compatibility between $\mathrm{ABC} / 2$ and computer-assisted SAS, irrespective of haematoma shape and poor approximation using modified ABC/2.

There are several potential explanations for variation in interobserver performance. Thinner slices may produce more accurate volume measurement, ${ }^{33} 34$ although the scans were identical for each observer. Alternatively, each additional slice to measure increases the potential for errors. Furthermore, observers may have chosen slightly different window settings. Additionally, although the observers were all trained in using OsiriX, accuracy in measurement does depend on operator experience. ${ }^{33} 36$

When comparing haemorrhage size on the basis of its longest measured diameter ('A' in $\mathrm{ABC} / 2$ ) with an adjudicated visual categorisation, there was 'strong' agreement, within the adjudicators and between the observers and adjudicators, with ICC 0.72 . Hence, it is unsurprising that the visual category versus the largest measured axial diameter has a slightly lower agreement than some of the other measured values. Note that the visual categorisation assigns an ordinal value based on the largest size in any plane, not just the axial plane, and thus is not the same as a linear measure of largest axial diameter.

Our study also shows that qualitative descriptors of haemorrhage characteristics of haemorrhage shape, density, presence of
Figure 5 Box plots of intracerebral haemorrhage $(\mathrm{ICH})$ volumes $(\mathrm{n}=548)$ by visually assessed shape and corresponding volume assessed by $A B C / 2$, modified $A B C / 2$ and semiautomatic segmentation (SAS) show: (1) larger ICHs are irregular in shape $(p<0.0001)$; $(2)$ the mean difference between modified $A B C / 2$ versus standard $A B C / 2$ and modified $A B C / 2$ versus $S A S$ increased as the haematoma shape became more difference was observed between standard $A B C / 2$ and SAS. irregular $(p<0.0001)$. No significant

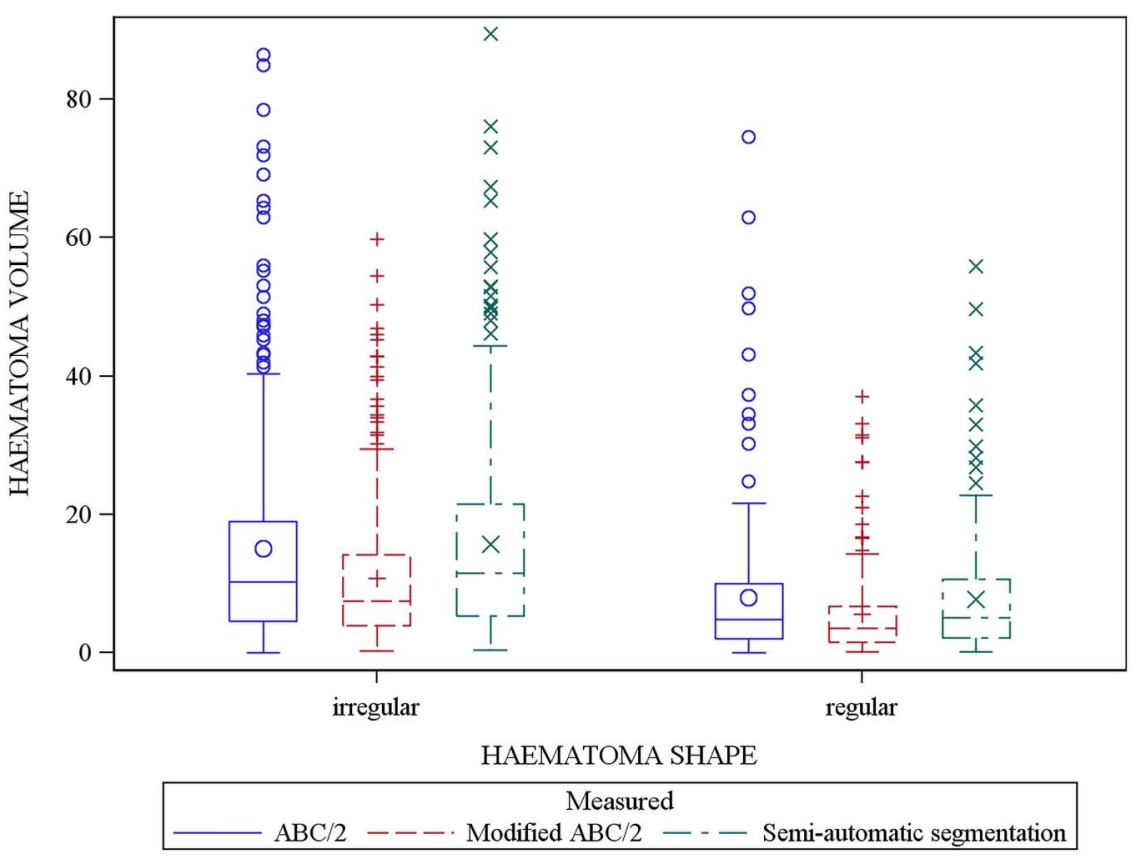


IVH using the Graeb and modified Graeb scores can be reliably measured and are reproducible.

Of note, when measuring density heterogeneity, we used only the coefficient of variation (cv), whereas Barras used four additional measures (although these were apparently inferior to cv). ${ }^{23}$ Calculation of shape index using parameters obtained from the windowed haemorrhage had a low ICC of 0.53 . When this result is held in context with similar computed volumes, it could be interpreted that the observer chose different threshold settings which explain the source of variability.

This study has several strengths including the use of multiple assessors, multiple methods for assessing haemorrhage characteristics, and a large data set. To minimise measurement error, observers were trained to use the OsiriX software for measuring brain CT scan parameters and in recognition of haemorrhages. Similarly, CT adjudicators were experienced neuroradiologists. Multiple observers were used to allow measurement of intraobserver and interobserver variation. The data set involved patients from five continents and so the findings have excellent external validity.

Nevertheless, the study has three significant limitations. First, the data come from a randomised controlled trial in acute stroke. Trial exclusion criteria can limit the type of patients (and therefore variation in haemorrhage) so that the data set studied here did not include patients with normal/low blood pressure, GCS $<8$, or without motor signs. As a result, patients with very large haematoma were under-represented. Second, the time from stroke onset to neuroimaging was relatively long (median $4.2 \mathrm{~h}$ ), reflecting that the ENOS protocol allowed enrolment up to $48 \mathrm{~h}$ after stroke. Hence, ICH on later scans may already have developed some peri-ICH oedema. Finally, just over $25 \%$ of the patients had measurable IVH, and therefore the results do not represent a population where IVH is more frequent. The failure to get a fully automated volume measure in $95 \%$ of scans may be less of a problem with other software that can handle scans of variable slice thickness.

In conclusion, the modified $\mathrm{ABC} / 2$ formula significantly underestimates haemorrhage volume when compared to standard $\mathrm{ABC} /$ 2 and computer assisted semiautomated segmentation (SAS) the difference increased as haematoma volume became larger and more irregular in shape. Most relevant for clinicians, our results show that the standard $\mathrm{ABC} / 2$ method offers more accurate quantification of ICH volume by the bedside. Although SAS is consistent and reliable, the method is slow, reliant on manual correction, advanced software and likely to cause delay in clinical decision-making often in emergency situations. Further research is required to create a faster computerised volumetric model for accurate and reliable measurement of haematomas in the clinical setting. Visual assessment using an ordered categorical scale has the strength of providing an 'instant' marker of haemorrhage size in the acute situation and can be applied in the absence of measurement tools and historical cut film data, and where very large study sizes or very moved images preclude computer based ABC/ 2 or SAS measurements. This study importantly shows that haemorrhage characteristics can be reliably measured as accuracy is pivotal to clinical trials in which ICH volume change may be a surrogate end point.

\footnotetext{
Author affiliations

${ }^{1}$ Stroke, Division of Clinical Neuroscience, University of Nottingham, Nottingham, UK ${ }^{2}$ School of Pathology and Laboratory Medicine, The University of Western Australia, Nedlands, Australia

${ }^{3}$ Department of Medical Imaging, College of Medicine, The University of Arizona, Arizona, USA

${ }^{4}$ Radiological Sciences Research Group, Division of Clinical Neuroscience, University of Nottingham, Nottingham, UK
}

${ }^{5}$ Department of Neuroradiology, Nottingham University Hospitals, Queen's Medical Centre, Nottingham, UK

${ }^{6}$ Stroke Centre, Ospedale Sacro Cuore Via Sempreboni, Verona, Italy

${ }^{7}$ Division of Neuroimaging Sciences, Centre for Clinical Brain Sciences, Western General Hospital, Edinburgh, UK

Correction notice Since this paper was first published online Lesley A Cala and Jennifer $L$ Becker have been added as authors.

Acknowledgements The authors thank the investigators and patients who participated in ENOS.

Funding ENOS was funded by the UK BUPA Foundation and Medical Research Council (G0501797). PMWB is The Stroke Association Professor of Stroke Medicine. JMW received funding from the Scottish Funding Council and Chief Scientist Office SINAPSE Collaboration (Scottish Imaging Network, A Platform for Scientific Excellence, http://www.sinapse.ac.uk).

Contributors The study was conceived by KK and PMB with imaging guidance led by JMW. KK wrote and prepared the first draft of the manuscript with input from the writing committee, all of whom approve for the final version of the manuscript to submitted and agree to be accountable for all aspects of the work undertaken in this research project.

\section{Competing interests None declared.}

Ethics approval UK and local research ethics committees in each participating country.

Provenance and peer review Not commissioned; externally peer reviewed.

Open Access This is an Open Access article distributed in accordance with the terms of the Creative Commons Attribution (CC BY 4.0) license, which permits others to distribute, remix, adapt and build upon this work, for commercial use, provided the original work is properly cited. See: http://creativecommons.org/ licenses/by/4.0/

\section{REFERENCES}

1 Broderick JP, Brott TG, Duldner JE, et al. Volume of intracerebral hemorrhage. A powerful and easy-to-use predictor of 30-day mortality. Stroke 1993;24: 987-93.

2 Wada R, Aviv RI, Fox AJ, et al. CT angiography "spot sign" predicts hematoma expansion in acute intracerebral hemorrhage. Stroke 2007:38:1257-62.

3 Almandoz JED, Yoo AJ, Stone MJ, et al. Systematic characterization of the computed tomography angiography spot sign in primary intracerebral hemorrhage identifies patients at highest risk for hematoma expansion the spot sign score. Stroke 2009;40:2994-3000.

4 Mendelow AD, Gregson BA, Fernandes HM, et al. Early surgery versus initial conservative treatment in patients with spontaneous supratentorial intracerebral haematomas in the International Surgical Trial in Intracerebral Haemorrhage (STICH): a randomised trial. Lancet 2005;365:387-97.

5 Mayer SA, Brun NC, Broderick J, et al. Safety and feasibility of recombinant factor VIla for acute intracerebral hemorrhage. Stroke 2005;36:74-9.

6 Lees KR, Bath PMW, Schellinger PD, et al. Contemporary outcome measures in acute stroke research: choice of primary outcome measure. Stroke 2012:43:1163-70.

7 Anderson CS, Huang Y, Wang JG, et al. Intensive blood pressure reduction in acute cerebral haemorrhage trial (INTERACT): a randomised pilot trial. Lancet Neurol 2008;7:391-9.

8 Mayer SA, Brun NC, Begtrup K, et al. Efficacy and safety of recombinant activated factor VII for acute intracerebral hemorrhage. N Engl J Med 2008;358:2127-37.

9 Kwak R, Kadoya S, Suzuki T. Factors affecting the prognosis in thalamic hemorrhage. Stroke 1983;14:493-500.

10 Kothari RU, Brott T, Broderick JP, et al. The ABCs of measuring intracerebral hemorrhage volumes. Stroke 1996;27:1304-5.

11 Gebel JM, Sila CA, Sloan MA, et al. Comparison of the ABC/2 estimation technique to computer-assisted volumetric analysis of intraparenchymal and subdural hematomas complicating the GUSTO-1 trial. Stroke 1998;29: 1799-801.

12 Huttner $H B$, Steiner $T$, Hartmann $M$, et al. Comparison of $A B C / 2$ estimation technique to computer-assisted planimetric analysis in warfarin-related intracerebral parenchymal hemorrhage. Stroke 2006;37:404-8.

13 Freeman WD, Barrett KM, Bestic JM, et al. Computer-assisted volumetric analysis compared with $A B C / 2$ method for assessing warfarin-related intracranial hemorrhage volumes. Neurocrit Care 2008;9:307-12.

14 Sheth KN, Cushing TA, Wendell $L$, et al. Comparison of hematoma shape and volume estimates in warfarin versus non-warfarin-related intracerebral hemorrhage. Neurocrit Care 2010;12:30-4

15 Barras CD, Tress BM, Christensen S, et al. Density and shape as CT predictors of intracerebral hemorrhage growth. Stroke 2009;40:1325-31. 
16 Graeb DA, Robertson WD, Lapointe JS, et al. Computed tomographic diagnosis of intraventricular hemorrhage- etiology and prognosis. Radiology 1982;143:91-6.

17 Morgan T, Dawson J, Spengler D, et al. The modified Graeb score an enhanced tool for intraventricular hemorrhage measurement and prediction of functional outcome. Stroke 2013;44:635-41.

18 The ENOS Trial Investigators. Glyceryl trinitrate vs. control, and continuing vs. stopping temporarily prior antihypertensive therapy, in acute stroke: rationale and design of the Efficacy of Nitric Oxide in Stroke (ENOS) trial (ISRCTN99414122). Int J Stroke 2006;1:245-9.

19 Wardlaw JM, Sellar R. A simple practical classification of cerebral infarcts on CT and its interobserver reliability. Am J Neuroradiol 1994;15:1933-9.

20 Sandercock P, Lindley R, Wardlaw J, et al. Third international stroke trial (ist-3) of thrombolysis for acute ischaemic stroke. Trials 2008;9:37.

21 Rosset A, Spadola L, Ratib O. OsiriX: an open-source software for navigating in multidimensional DICOM images. J Digit Imaging 2004;17:205-16.

22 Pabst W, Gregerova E. Characterisation of particle and particle systems. Prague: ICT, 2007;27-29.

23 Barras CD, Tress BM, Christensen S, et al. Quantitative CT densitometry for predicting intracerebral hemorrhage growth. Am I Neuroradiol 2013;34:1138-44.

24 Shrout P, Fleiss J. Intraclass correlations: uses in assessing rater reliability. Psychol Bull 1979;86:420-8.

25 ENOS Investigators. Baseline characteristics of the 4011 patients recruited into the "efficacy of Nitric Oxide in Stroke (ENOS) trial. Int I Stroke 2014;9:711-20.

26 Bath PMW, Woodhouse L, Scutt P, et al. Management of high blood pressure in acute stroke: efficacy of Nitric Oxide in Stroke (ENOS), a partial-factorial randomised controlled trial. Lancet 2014. Published online 22 Oct 2014 http://dx.doi.org/ 10.1016/ s0140-6736(14)61121-1
27 Wang CW, Juan CJ, Liu YJ, et al. Volume-dependent overestimation of spontaneous intracerebral hematoma volume by the ABC/2 formula. Acta Radiol 2009;50:306-11.

28 Maeda AK, Aguiar LZ, Martins C, et al. Hematoma volumes of spontaneous intracerebral hemorrhage: the ellipse $(\mathrm{ABC} / 2)$ method yielded volumes smaller than those measured using the planimetric method. Arquivod de Neuro-Psiquiatria 2013;71:540-4

29 Yang $W$, Feng $Y$, Zhang $Y$, et al. Volume quantification of acute infratentorial hemorrhage with computed tomography: validation of the formula $1 / 2 \mathrm{ABC}$ and $2 /$ 3SH. PLOS ONE 2013;8:e62286.

30 Zhao KJ, Liu Y, Zhang RY, et al. A precise, simple, convenient and new method for estimation of intracranial hematoma volume-the formula 2/3sh. Neurol Res 2009;31:1031-6.

31 Sucu HK, Gokmen M, Gelal F. The value of XYZ/2 technique compared with computer-assisted volumetric analysis to estimate the volume of chronic subdural hematoma. Stroke 2005;36:998-1000.

32 Brott T, Broderick J, Kothari R, et al. Early hemorrhage growth in patients with intracerebral hemorrhage. Stroke 1997;28:1-5.

33 Divani AA, Majidi S, Luo $X$, et al. The ABCs of accurate volumetric measurement of cerebral hematoma. Stroke 2011;42:1569-74.

34 Prionas ND, Ray S, Boone JM. Volume assessment accuracy in computed tomography: a phantom study. J App/ Clin Med Phys 2010;11:3037.

35 Kosior J, Idris S, Dowlatshahi D, et al. Quantomo: validation of a computer-assisted methodology for the volumetric analysis of intracerebral haemorrhage. Int I Stroke 2011;6:302-5.

36 Hussein HM, Tariq NA, Palesch YY, et al. Reliability of hematoma volume measurement at local sites in a Multicenter Acute Intracerebral Hemorrhage Clinical Trial. Stroke 2013;44:237-9. 